\title{
BJMHR
}

British Journal of Medical and Health Research

Journal home page: www.bjmhr.com

\section{CRISPR-CAS9 Technology Mechanism- The New Gene Editing Tool for Disease Prevention \& Treatment.}

\author{
R. H. Mishal ${ }^{1}$, B. H. Mishal ${ }^{2}$, H. B. Mishal $^{3}$ \\ 1. Dr. M. S. Gosavi College of Pharmaceutical Education \& Research, Prin. T. A. Kulkarni \\ Vidyanagar, Nashik-422005. \\ 2.Sinhgad Institute's Sinhgad College of engineering, Vadgaon (Bk), Pune-411041. \\ 3.MET's Institute of Pharmacy, Bhujbal Knowledge City, Adgaon, Nashik-422003.
}

\section{ABSTRACT}

Genome editing (also called gene editing) is a group of technologies that give scientists the ability to change an organism's DNA. These technologies allow genetic material to be added, removed, or altered at particular locations in the genome. Several approaches to genome editing have been developed. A recent one is known as CRISPR-Cas9, which is short for clustered regularly interspaced short palindromic repeats and CRISPR-associated protein 9. The CRISPR-Cas9 system has generated a lot of excitement in the scientific community because it is faster, cheaper, more accurate, and more efficient than other existing genome editing methods. Genome editing is of great interest in the prevention and treatment of human diseases. Currently, most research on genome editing is done to understand diseases using cells and animal models. Scientists are still working to determine whether this approach is safe and effective for use in people. It is being explored in research on a wide variety of diseases, including single-gene disorders such as cystic fibrosis, hemophilia, and sickle cell disease. It also holds promise for the treatment and prevention of more complex diseases, such as cancer, heart disease, mental illness, and human immunodeficiency virus (HIV) infection. This paper throws light on this technology.

Keywords: CRISPR, DNA sequence, genome editing, gene therapy, immune system. 


\section{INTRODUCTION}

CRISPR is a family of DNA sequences in bacteria. The sequences contain snippets of DNA from viruses that have attacked the bacterium. These snippets are used by the bacterium to detect and destroy DNA from similar viruses during subsequent attacks. These sequences play a key role in a bacterial defence system and form the basis of a technology known as CRISPR/Cas9 that effectively and specifically changes genes within organisms. The CRISPR/Cas system is a prokaryotic immune system that confers resistance to foreign genetic elements such as those present within plasmids and phages that provides a form of acquired immunity. In bacteria and archaea CRISPR-Cas9 was discovered as an acquired immune system against viruses and phages. CRISPR/Cas9 is observed in nearly $40 \%$ genomes of sequenced bacteria and 90\% genomes of sequenced archaea. Being the most abundant entities on our planet, bacterial and archaeal viruses display a constant threat to prokaryotic life. In order to withstand these threats prokaryotes have evolved different defense strategies.CRISPR-Cas9 is one such defense mechanism evolved by the prokaryotes. It is regarded as unique because of its adaptive nature and therapeutic potential. CRISPR/Cas9 genome editing techniques have many potential applications, including medicine and crop seed enhancement. In this mechanism small guide RNAs (crRNAs) are employed for sequence specific interference with invading nucleic acids. CRISPR-Cas associated with Cas protein 9 system has a genomic locus with short repeats separated by unique spacers ${ }^{1}$. These are originating from mobile genetic elements (MGEs) like bacteriophages, transposons, plasmids. This technology depends on small RNA for sequence specific cleavage. In CRISPR-Cas9 technology invading foreign DNA segments are processed Cas nuclease into small fragments. Subsequent DNA repair processes leads to desired insertions, deletions or substitutions at target sites.

This technology is a very innovative, unique genome editing tool. The viral snippets introduced in the bacterial genome can provide immunological advantage to the bacteria.

\section{Molecular Mechanism: Adaptations, Maturation, Interference:}

\section{Adaptation:}

In the first phase, a distinct invading MGE sequence is introduced as a protopacer in the CRISPR locus yielding a new spacer. This helps the host to memorize the invader's genetic material. This displays the adaptive nature of the host Immune system. The selection of a target sequence is not random.

\section{Biogenesis:}

To enable immunity, the CRISPR array is transcribed into a long precursor crRNA that is further processed into mature guide crRNAs containing the memorized sequences of the 
invaders. Cas9 is a endoribonuclease that generates guide RNAs for invader defense in prokaryotes sequence and structure specific RNA processing by a CRISPR (endonuclease). More than 40 different Cas protein families have been reported. A guild of 45 CRISPRassociated Cas protein families and multiple CRISPR/Cas subtypes exist in prokaryotic genomes playing an important role in crRNA biogenesis, spacer incorporation and invading DNA cleavage.

1. Based on the sequences and Cas protein structures they are classified into three types, I,II and III

\section{Interference:}

In the last stage of immunity, mature crRNAs are used as guides to specifically interfere with the invading nucleic acids ${ }^{2,3}$. In endogenous CRISPR/Cas9 system, mature crRNA is combined with trans activating crRNA (tracrRNA) to form a tracrRNA:crRNA complex that guides Cas9 to target site. TracrRNA is partially complementary to crRNA and contributes to crRNA maturation. At the target site, CRISPR/Cas9 -mediated sequence -specific cleavage requires a DNA sequence protospacer matching crRNA and a shorter protospacer adjacent motif(PAM).

3. For easy application of genome editing, researchers designed a delicate guide RNA (gRNA).

\section{Applications:}

\section{Genome editing:}

CRISPR/Cas9 provides a robust, multiplexable genome editing tool. This technology helps us in manipulating specific genomic elements and for determination of functions and its elucidation in of target genes in diseases and biology ${ }^{4}$. CRISPR/cas9 is also used to induce specific modifications in human genome of cells.

This technology helps us to introduce mutations in certain genes. The generated mutations are transmitted to the further generations through the germ line.

\section{Transcription regulation:}

Gene transcription regulation in living organisms is very useful for gene function and transcriptional network studies. This technology can also be used for the regulation of transcription of certain specific genes. This process of transcription is irreversible due to permanent DNA modifications.

\section{Gene therapy:}

Genome editing can also be used to permanently cure certain diseases. This technology may be used for disrupting endogenous disease causing genes, correcting disease-causing 
mutations or inserting new protective genes for the prevention of diseases like cancer, diabetes, etc.

\section{Significance:}

The use of CRISPR/Cas9 in therapeutic approaches has become increasingly relevant in different fields of medicine. It is also used in the treatment of various diseases. Certain genetic diseases can be cured by modifications of genes or eliminating certain disease causing genes. It is most importantly used as a genome editing tool. For various therapeutic purposes CRISPR/Cas9 technology can be used.

\section{Challenges:}

\section{Off-target mutations:}

Off-target mutations are one major concern about CRISPR/Cas9-mediated genome editing. Large genomes often contain multiple DNA sequences identical that are identical or highly homologous DNA sequences, which leads to mutations at undesired sites, called off-target mutations. To reduce the cellular toxicity these off-target mutations should be reduced.

\section{PAM Dependence:}

Theoretically, CRISPR/Cas9 can be applied to any DNA sequence through engineered programmable gRNA. The dentified PAM sequences vary among different Cas 9 orthologs, such as NGG PAM from Streptococcus pyro genes.

\section{gRNA production:}

gRNA is a guiding RNA sequence. It is one of the important issue in CRISPR/Cas9 technology.

\section{Perspectives:}

Interest in CRISPR technology has been rapidly increased in these recent years. Numerous studies explain the genetic and biochemical processes involved in the immune system of the prokaryotes 5 . This technology is widely used because of its medicinal applications. This technology also has biotechnological appications like crop editing, gene drives and synthetic drives.

\section{CONCLUSION:}

The development of efficient and reliable ways to make precise, targeted changes to the genome of living cells is a long-standing goal for biomedical researchers. CRISPR/Cas9 technology is a powerful genome editing tool. It is expected ideally that a genome editing tool should be efficient, inexpensive, and simple. It should also avoid off-target mutations. We can say that CRISPR/Cas9 is a reliable genome editing tool since it almost has all the features mentioned above. The rapid progress in developing Cas9 into a set of tools for cell and molecular biology research has been remarkable, likely due to the simplicity, high 
efficiency and versatility of the system. Of the designer nuclease systems currently available for precision genome engineering, the CRISPR/Cas system is by far the most user friendly. Hence this technology can be used for wide range of applications.

\section{REFERENCES:}

1. CRISPR/Cas9 for Genome Editing: Progrss, implications \& challenges; Xiong Guo et al; Human Molecular Genetics; 2014; 23(01); R40- R46.

2. Genome Engineering through CRISPR/Cas9 Technology in the Human Germline and Pluripotent Stem Cells; R. Vassena et al; Human Reproduction Update; 2016; 22(04); pp 411-419.

3. Genome Engineering Using the CRISPR-Cas9 System; F Ann Ran et al; Nature Protocols; 2013; 08(11); pp 2281- 2308.

4. CRISPR-Cas: Biology, Mechanisms and Relevance; Frank Hille et al; The Royal Society Publishing; 2016.

5. Genome Editing: The Road of CRISPR/Cas9 From Bench to Clinic; Ayman Eid et al; Experimental and Molecular Medicine (2016) 48; pp 01-11.

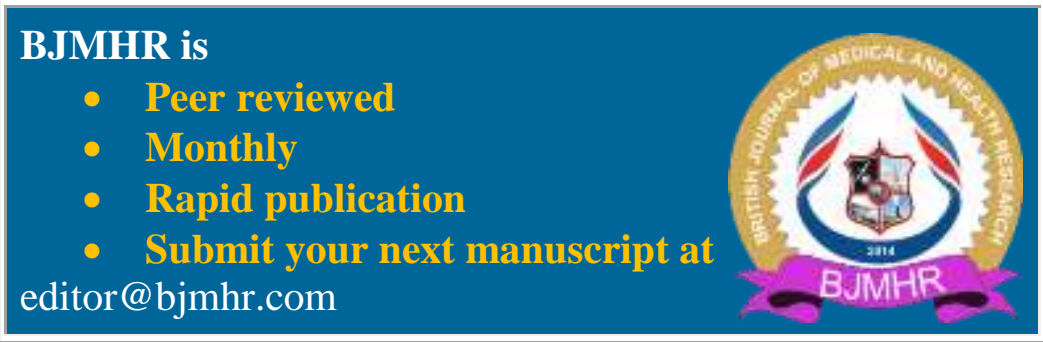

